\title{
Pre-Thinking GIS - Zur Visuellen Politik der frühen quantitativ-theoretischen Geographie
}

\author{
Boris Michel \\ Universität Erlangen-Nürnberg, Institut für Geographie, Wetterkreuz 15, 91058 Erlangen, Germany \\ Correspondence to: Boris Michel (boris.michel@fau.de)
}

Received: 24 November 2016 - Revised: 12 July 2017 - Accepted: 9 August 2017 - Published: 7 September 2017

\begin{abstract}
Kurzfassung. This paper examines questions of how issues of visual representation and vision have changed with the the quantitative and theoretical thinking in 1950s and early 1960s Anglophone geography. If the quantitative revolution in geography is understood as a scientific revolution, one should also expect a revolution of the ways in which geography made use of visualizations. At the center of this essay is William Bunge's "Theoretical Geography", one of the founding text of this new geographical thought. This book forms the starting point for a discussion of the changed roles and changed forms of visualization in the production of geographical knowledge. Following Fred Schaefer's attack on Richard Harthorne, Bunge placed the search for morphological laws at the center of a geography that is strongly oriented towards geometry. In this paper, the text of Bunge serves as a starting point into the field of early analytical cartography and the first consideration of what later, become geographic information systems and their new visual language. In this paper this history of GIS is largely told without a history of technology a well as without its political context of the cold war and the Fordist state.
\end{abstract}

\section{Einleitung}

Geographische Expeditionen und Forschungsreisen im 19. Jahrhundert wurden vielfach von Malern und professionellen Photographen begleitet, die dafür Sorge zu tragen hatten, dass der visuelle Eindruck der erforschten Landschaft in naturwahrer Form aus der Ferne in Wissensbestände der europäischen Universitäten gebracht wurde. Später wurden diese durch handliche Fotoapparate ersetzt und durch eine universitäre Ausbildung, in der das Abzeichnen der Landschaft und Kartieren als zentrale Techniken der Disziplin gelehrt wurden. Ende der 1960er Jahre schreiben Haggett und Chorley, im Anschluss an die kartographische Darstellung eines Flusssystems, dass dieser Anblick auch übersetzt werden könne in die Formulierung „1110110010100111000010100“ (Haggett und Chorley, 1969:10). Zwischen diesen beiden Darstellungen liegt das, was man zumeist als einen Paradigmenwechsel bezeichnet und in diesem Fall ein Paradigmenwechsel, der sich der mathematischen Sprache, insbesondere in Form von Geometrie, verschrieben hat. Wissenschaftliche Disziplinen und Paradigmen verfügen nicht nur über eine eigene Sprache und narrative und technische Verfahren der Produktion von legitimen Aussagen, sondern auch über ein eigenes Sehen und Darstellen. Was sichtbar ist und wie „epistemischen Dingen“ (Rheinberger, 2002:24) ein Bild gegeben wird, steht in Verbindung zu den jeweiligen wissenschaftstheoretischen Annahmen, epistemischen Tugenden und Praktiken einer Disziplin. Wissenschaftliche Paradigmen produzieren unterschiedliche „Weltsichten“, eine Feststellung, die Thomas Kuhn sowohl metaphorisch wie auch wörtlich meinte (Kuhn, 1973:123f). In Folge hat sich in der neueren Wissenschaftsgeschichte und Wissenschaftsforschung ein umfangreiches Interesse an Praktiken, Rationalitäten und Diskursen um Visualisierung und Visualität entwickelt, die von Foucault Genealogie des medizinischen Blicks über Arbeiten aus dem Feld von ANT und historischer Epistemologie bis zu aktuellen STS reichen (Michel, 2013).

Dieser Aufsatz befasst sich aus einer wissenschaftsgeschichtlichen Perspektive mit dem Visualitätsregime der Geographie der quantitativ-theoretischen Wende, also jener Bewegung in der Geographie, die 1962, als Kuhn „The Structure of Scientific Revolutions" erstmals veröffentlichte, ihren ersten Zenit bereits erreicht hatte (Burton, 1963). Es wird der Frage nachgegangen, wie sich im Denken wesentlicher Vertreter der quantitativ-theoretischen Wende der anglophonen 
Geographie Fragen der Darstellung und des Sehens geändert haben und welche Rolle diesen Verfahren in methodologischen und methodischen Überlegungen zugeschrieben wurde. Wenn die Quantitative Revolution eine wissenschaftliche Revolution war und wissenschaftliche Revolutionen, wie Kuhn oder auch Fleck in ihren wissenschaftsgeschichtlichen Klassikern deutlich gemacht haben, auch Sichtbarkeit und Aussehen der wissenschaftlichen Gegenstände transformieren (Kuhn, 1973:123f; Fleck, 1980), dann ist auch eine Revolutionierung des geographischen Visualitätsregime zu erwarten (Michel, 2015). Im Zentrum dieses Aufsatzes steht dabei zunächst William Bunges „Theoretical Geography“ (Bunge, 1962). Der Text bildet den Ausgangspunkt einer Diskussion zur veränderten Rolle und veränderten Form von Visualisierung in der geographischen Wissensproduktion. Im Anschluss an Fred Schaefer und dessen einflussreichen Text „Exceptionalism in Geography“ (Schaefer, 1953) hat Bunge die Suche nach morphologischen Gesetzen ins Zentrum einer Geographie gerückt, die sich damit stark an der Geometrie orientiert. Der Text von Bunge dient in diesem Aufsatz als Sprungbrett in das Feld der frühen analytischen Kartographie und zu ersten Überlegungen zu dem, was später als Geographische Informationssysteme (GIS) eine neue Weise der visuellen Sprache für die Geographie ermöglichte.

Die Geschichte von GIS ist in den letzten Jahren verstärkt in den Blick geographischer Disziplingeschichtsschreibung gerückt. In den meisten dieser Arbeiten wird insbesondere und aus guten Gründen eine Geschichte der Technik, der Institutionen oder des gesellschaftlichen Kontexts von GIS geschrieben (Wilson, 2014; Barnes, 2013; Chrisman, 2006; Barnes und Wilson, 2014; Rankin, 2016). Dahingegen will dieser Text eine stärker epistemologisch ausgerichtete Vorgeschichte von GIS schreiben, die weitgehend ohne Computer auskommt. Wichtiger als die technischen Instrumente digitaler Datenverarbeitung oder der militärisch-industriellakademische Komplex des Kalten Kriegs sind in diesem Aufsatz daher Momente geographischen Denkens, die es für die Geographie erst plausibel gemacht haben, Digitales, Daten und rechnerische Verarbeitung für etwas zu halten, das relevant und sinnhaft für die Wissensproduktion der Disziplin ist. Damit wird natürlich nicht die Bedeutung jener Instrumente, Maschinen und Praktiken geleugnet, ebenso wenig die Rolle des außerwissenschaftlichen Kontexts, diese werden jedoch unter anderem von den oben genannten AutorInnen ausführlich thematisiert.

Der Text von Bunge dient dabei als Verdichtung und Synthese einer entstehenden Debatte, bringt er doch wie kein anderer Text dieser Zeit einen Großteil der neuen Literatur in einen kohärenten Rahmen. Zunächst sollen aber kurz und sehr schematisch einige wesentliche Momente der quantitativ-theoretischen Wende rekapituliert werden.

\section{Die quantitativ-theoretische Wende in der Anglophonen Geographie}

Der Begriff der Quantitativen Revolution ist, darauf haben bereits zeitgenössische Autoren hingewiesen (Burton, 1963; Bartels, 1968:12; Gould, 1979:140), irreführend, impliziert er doch eine Reduzierung auf quantitative Verfahren. Dies lenke davon ab, dass es sich aber um einen umfassenden Paradigmenwechsel handelte. ,It was not the numbers that were important, but a whole new way of looking at things" (Gould, 1979:140). Der Konflikt zwischen alter und neuer Geographie, so Harvey, habe jedoch ,nothing to do with whether or not one employs a chi-square test or calculates a regression line, but [is a conflict] between two views of what are tractable and interesting geographical questions, and what are satisfying and reasonable answers" (Harvey, 1969:17).

Gegenüber Geographie als Länderkunde und in Abgrenzung zu Autoren wie Alfred Hettner, Richard Hartshorne oder Carl Sauer, beginnt sich seit den frühen 1950er Jahren in der anglophonen Geographie ein neues Modelldenken zu etablieren. Mit dessen Bezugnahme auf wissenschaftstheoretische Ansätze wie logischen Positivismus und später auch auf kritischen Rationalismus ${ }^{1}$ schließt die Geographie an eine allgemeine Bewegung hin zu einer Mathematisierung und Technisierung der modernen Wissenschaften an. Gegenüber der seit Immanuel Kant immer wieder proklamierten Sonderstellung der Geographie im System der Wissenschaften wird gefordert, die wissenschaftstheoretische Grundlage der Disziplin im Sinne einer einheitswissenschaftlichen Rationalität zu fundieren (Schaefer, 1953). Gegen eine Wissenschaft, die in der Beschreibung und unmittelbaren Beobachtung gründet, tritt - zumindest bei den stärker wissenschaftstheoretisch interessierten Autoren - ein Primat von Theorie, Problemorientierung und Kalkulation. In den Augen von Harvey und anderen vollzieht die Geographie damit im Feld der Wissenschaften eine nachholende Revolution (Harvey, 1969:65). Das Versprechen dieser Revolution ist nichts weniger, als aus der Geographie eine Wissenschaft zu machen und sie als einen ernstzunehmenden Teil der modernen akademischen

\footnotetext{
1 anders als in der deutschsprachigen Geographie, wie etwa bei Bartels (Bartels, 1968) und wie in Schilderungen der quantitativtheoretischen Geographie immer wieder impliziert, ist eine explizite Bezugnahme auf Popper und den kritischen Rationalismus in den frühen Beiträgen der anglophonen Geographie selten. Eine intensive Auseinandersetzung mit zeitgenössischer Wissenschaftstheorie in Form umfangreicherer Veröffentlichungen hat vor Harveys „Explanation in Geography“ (Harvey, 1969) überraschenderweise kaum stattgefunden. Die meisten der hier erwähnten Arbeiten der quantitativ-theoretischen Geographie geben wenig Auskunft über wissenschaftstheoretische Bezüge, die jenseits einer allgemeinen Gleichsetzung von Wissenschaftlichkeit mit Mathematisierung und Theorie liegen. Siehe beispielsweise die einleitenden Seiten von Bunges „Theoretical Geography“, die lediglich einige wenige Bezüge auf den logischen Positivismus des Wiener Kreises herstellen (Bunge, 1962)
} 
Arbeitsteilung zu begründen, etwas was im Anschluss an den 2. Weltkrieg sowie der Schließung der Geographie in Harvard als bedroht angesehen wurde (Ackerman, 1945; Smith, 1987).

Historisch ist diese quantitativ-theoretische Geographie eng verbunden mit dem militärisch-industriell-akademischen Komplex am Ende des Zweiten Weltkriegs. Ihre Etablierung ist nicht ohne Bezug auf den Kalten Krieg und die veränderten Praktiken und Diskurse staatlicher Raumordnungspolitiken zu verstehen. Der Einsatz neuer Technologien, ein gewandelter institutioneller Rahmen universitärer und außeruniversitärer Forschung, neue Wissenschaften wie die Kybernetik und Systemtheorie, oder wissenschaftsoptimistische Modernisierungstheorien (Erickson et al., 2013:4; Murphy, 2016; Solovey und Cravens, 2012), prägten, ob implizit oder explizit, den intellektuellen Rahmen, in dem einzelne Geographen $^{2}$ an einigen Orten begannen, eine neue Geographie zu denken, zu praktizieren und institutionell durchzusetzen. Dieser erweiterte gesellschaftliche und intellektuelle Kontext geht auch einher mit neuen Formen und Funktionen visuellen Denkens und Argumentierens in den unterschiedlichsten akademischen und gesellschaftlichen Feldern (Halpern, 2014; Hagner, 2006; Barney, 2015).

Zwar befreit sich die Geographie mit der quantitativtheoretischen Wende zunächst von Konzepten wie Landschaft und damit von einer Geographie, die in der Beschreibung der sichtbaren Dinge im Raum ein wesentliches Ziel sieht. Diese „Neue Geographie“ war aber alles andere als visualisierungsskeptisch. Vielmehr macht bereits ein kursorischer Blick deutlich, dass eine Vervielfältigung von visuellen Darstellungen stattfindet (Michel, 2016a). Auch nimmt, trotz einer Rhetorik der Nüchternheit, des Kalküls und eines mathematischen Formalismus', visuelle Metaphorik in den Texten keinesfalls ab. Nur sind es statt Momenten des Erhabenen, der dichten Schilderung und der Naturschönheit der klassischen Geographie nun Metaphern aus Mathematik, Physik und Biologie und statt Fotografien räumlicher Individuen nun Visualisierungen wie Walter Christallers Hexagone der zentralen Orte. Dass ein Paradigma, das sich einer Mathematisierung des Denkens, der Abstraktion, Gesetzen und der Prognostik verschrieben hat, eine größere Affinität zur Visualisierung geographischer Sachverhalte aufweist, als eines, das sich dem Konkreten, dem Individuellen und der Deskription verbunden sieht, mag auf den ersten Blick verwundern. Dennoch weisen gerade methodologische und theoretische Arbeiten der quantitativ-theoretischen Geographie in einem Umfang visuelle Darstellungen auf, wie dies die Geographie vorher und nachher nicht kannte (Isard, 1956; Bunge,

\footnotetext{
${ }^{2}$ In der Tat sind alle der hier zitierten Titel der quantitativtheoretischen Geographie im Namen männlicher Autoren erschienen. Die männliche Schreibform zu verwenden, soll gerade das deutlich machen. Ausführlicher dazu: (Barnes, 2004:584; Hanson, 1993).
}

1962; Garrison und Marble, 1967; Haggett, 1965; Berry und Marble, 1968; Chorley und Haggett, 1967).

Nicht nur, weil wissenschaftliche Revolutionen meist spannender sind als die Mühen der akademischen Ebene, liegt der Fokus dieses Beitrages auf frühen Arbeiten einer quantitativ-theoretischen Geographie. Vielmehr bedeutet der Einbruch des Neuen in bestehende Strukturen in einer anderen Weise eine Begründungsnotwendigkeit, als dies für ein stabilisiertes Paradigma gilt. Einer der einflussreichsten und zugleich radikalsten Grundlegungsversuche dieser neuen Geographie war William Bunges ,Theoretical Geography“ (Bunge, 1962).

\section{Geometrie als die Sprache der räumlichen Form}

Nachdem Bunge in den USA keinen veröffentlichungswilligen Verlag gefunden hatte, erschien seine 1960 an der University of Washington in Seattle als Promotion angenommene Arbeit „,Theoretical Geography“ in deutlich überarbeiteter Form 1962 als erster Band der Reihe ,Lund Studies in Geography C. General and Mathematical Geography“ (Bunge, 1962) in Schweden. Die University of Washington war einer der maßgeblichen Orte, von denen aus sich die quantitativtheoretische Geographie ab den 1950er Jahren entwickelte (Barnes und Abrahamsson, 2017:115). Hier lehrten Garrison und Ullman seit den frühen 1950er Jahren. Mit Berry, Davey, Marble, Nystuen, Tobler, Getis und eben „Wild Bill““ Bunge studierten und arbeiten hier einflussreiche Vertreter der neuen Geographie. Durch zahlreiche Gastaufenthalte von Kollegen, war das Institut für Geographie wesentlicher Knotenpunkt im Netzwerk von Standorten, aus denen diese neue Geographie hervorging. Es war zudem eine der ersten Universitäten, an denen Geographen ab Mitte der 1950er Jahre mit einem Computer, einem neu angeschafften IBM 650, arbeiteten (Barnes, 2004, 2013).

Es ist nicht leicht die Bedeutung einzuschätzen, die ,Theoretical Geography“ für die Entwicklung dieser neuen Geographie hatte. Der Text gilt einerseits als Klassiker der quantitativ-theoretischen Geographie. Cox bezeichnet ihn als ,perhaps the seminal text of the spatial-quantitative revolution" (Cox, 2001:71), ein Attribut, das ansonsten auch Harveys „Explanation in Geography“ (Harvey, 1969) zugesprochen wird. In Form und Inhalt ist ,Theoretical Geography“ gewiss das „rebellischere“ und ,revolutionärere“ der beiden Bücher, markiert es doch eher einen Aufbruch und eine Aufforderung, als das später geschriebene und wissenschaftstheoretisch wesentlich elaboriertere Buch von Harvey. Zugleich wurde Bunges Arbeit, so Goodchild, vermutlich nicht oft gelesen und ist sicherlich ein sperriger Text, dessen direkter Einfluss schwer kenntlich zu machen ist (Goodchild, 2011; Cox, 2001). Gewiss wurde es seltener und weniger ausführlich besprochen als „Explanation in Geography“ und die Schwierigkeiten bei der Veröffentlichung weisen darauf hin, dass sich der Text zu Beginn der 1960er Jahre an der 
Grenze dessen bewegte, was als legitime Geographie galt. Beide Bücher jedoch, so Livingstone, machten ihre Autoren zu den ,godfathers at geography's positivist baptism“ (Livingstone, 1992:329). In den letzten Jahren haben sich zudem eine Reihe von wissenschaftsgeschichtlichen Arbeiten in der Geographie mit dem Leben und Arbeit von Bunge beschäftigt. Sei es in Form von Diskussionen (Barnes und Heynen, 2011; Merrifiled, 2011; Mountz, 2011) um die Neuauflage des Buches „Fitzgerald. Geography of a Revolution“ (Bunge, 2011), bezüglich seiner Rolle für die Kartographie im Kalten Krieg (Barney, 2015) oder in Form stärker biographisch orientierter Beiträge (Barnes, 2016, 2017).

Wie der Titel des Buches nahelegt, ist dessen Ziel alles andere als zurückhaltend, der ursprüngliche Arbeitstitel, so Bunge in einem Brief an Hartshorne, war gar ,Fundamental Geography“ (Bunge, 1959). ${ }^{3}$ Vielmehr wird beansprucht, einen ersten umfangreichen Beitrag zu einer umfassenden Neubestimmung der Geographie zu liefern und gegenüber einer deskriptiven Geographie eine Theorie der Geographie und eine geographische Theorie vorzulegen. Daher ist der Gestus der Arbeit vielfach proklamatorisch und es werden eine Vielzahl verbündeter Texte und Autoren der quantitativtheoretischen Geographie mobilisiert, die den Text als Teil einer größeren Bewegung verorten. Das Buch ist ein Manifest für eine neue Geographie, das sich wenig mit Nuancen aufhält und das im Vergleich zu Harvey weniger Bezug nimmt auf außergeographische Autoren aus Philosophie und Wissenschaftstheorie. Dafür bringt er erstmals eine große Zahl von Beiträgen eines neuen geographischen Denkens zwischen zwei Buchdeckeln zusammen.

Die neue Geographie, die Bunge vorschlägt, ist zunächst eine ,wissenschaftliche“ und eine ,theoretische“ Geographie. Wissenschaftlich bedeutet für ihn, dass diese positivistisch und nomothetisch ist und damit in Tradition des logischen Positivismus dem Standardmodell wissenschaftlicher Erklärung entspricht. Eine grundsätzliche Differenz zwischen sozialwissenschaftlichen, geisteswissenschaftlichen und naturwissenschaftlichen Methodologien lehnt Bunge ab, bzw. alle Wissenschaften werden der Methodologie der Naturwissenschaften angepasst. Immer wieder werden auch visuelle Analogien zu naturwissenschaftlichen Gesetzen gesucht um die eigene Suche nach geographischen Raumgesetzen zu unterstützen. Wesentlich für Bunge ist eine Mathematisierung geographischen Denkens. Daher beginnt das Buch mit relativ allgemeinen Überlegungen zu Funktion und Wesen von Theorie in den Wissenschaften und der Frage, wie eine solche Theorie auszusehen habe, wenn

\footnotetext{
${ }^{3}$ Der Briefwechsel zwischen Hartshorne und Bunge, der sich im Nachlass von Hartshorne im Archiv der AGS, Milwaukee, befindet, gehört sicherlich zu den kuriosesten Briefwechseln in der Geschichte der Geographie, ein Briefwechsel zwischen dem geduldigen $e l$ der statesman Hartshorne und einem in vielerlei Hinsicht wilden, radikalen und oft ziemlich unverschämten Bunge. Oder, wie Barnes in einem Text zum Verhältnis der beiden treffend schreibt, „It was Oedipus Rex and Monty Python joined“ (Barnes, 2016).
}

sie auf die Geographie übertragen wird. Im Zentrum stehen dabei zum einen Fragen der wissenschaftlichen Praxis der Regionalisierung in der Geographie und damit Fragen einer Modernisierung der länderkundlich regionalen Geographie zu einer Regional Science, wie es zuvor Isard gefordert hatte (Isard, 1960). Zum anderen werden Fragen der Beschreibung und Erklärung von Verteilungen und räumlichen Mustern als eine Reformulierung einer Allgemeinen Geographie diskutiert.

Das zweite Kapitel befasst sich mit Karten als dem wesentlichen Instrument und „logical framework“ (Bunge, 1962:33) geographischen Denkens. Unter dem Begriff der metacartography untersucht dieses Kapitel Möglichkeiten und Grenzen kartographischer Darstellung im Rahmen geographischer Theorie und überschreitet dabei explizit die traditionellen Fragen der Geographie an die Herstellung und den Gebrauch von Karten. Metakartographie, so Bunge, stellt die Frage, welche Möglichkeiten und Funktionen Karten als Instrumente zur Darstellung räumlicher Eigenschaften im Vergleich zu anderen Instrumenten wie Fotografien, Bildern, Graphen, Sprache oder Mathematik haben (Bunge, 1962:38). Während Bunge sich einerseits mit der Grenze zwischen Karten und pre-maps (Fotografien, Bildern, Graphen oder Sprache) befasst, und er letztere der Ebene reiner Beschreibung und damit einer traditionellen Geographie zuordnet, gilt das Hauptinteresse dem Verhältnis und der Übersetzung zwischen Karte und Mathematik und dabei einer Mathematisierung der Kartographie. Theoretische Geographie und Karten fungieren als wesentliches Moment der Vermittlung zwischen dem Konkreten und Abstrakten, zwischen Tatsachen und Logik, pre-maps und Mathematik. Genau diese vermittelnde Brückenfunktion zwischen Mathematik und der Welt der konkreten räumlichen Erscheinungen ist es, die Bunge der Geographie zuschreibt (Bunge, 1962:37ff).

Daran anschließend und die Überlegungen weiter in Richtung Mathematik und Geometrie treibend, befasst sich das folgende Kapitel mit der Messung von Formen und ,bridges the gap between maps and mathematics by demonstrating that the spatial property of shape can be converted into descriptive mathematics“ (Bunge, 1962:x). Hiermit wird angeregt, kartographische Repräsentationen in Form von Vektoren und Polygonen zu denken. Es folgen ein Kapitel zu deskriptiver Mathematik, das eine Art Einführung in die Statistik für Geographen ist, insbesondere in Hinblick auf räumliche Stichproben, sowie drei abschließende Kapitel in denen sich Bunge mit nichtdeskriptiver Mathematik in der Geographie beschäftigt. Letztere befassen sich mit zwei der erfolgreichsten Felder theoretischer Geographie der frühen 1960er Jahre. Mit einer general theory of movement für die Autoren wie Ullman und Warntz stehen sowie mit Theorien der zentralen Orte, in denen Bunge die bisher erfolgreichsten und konsistentesten Beiträge einer theoretischen Geographie sieht. Das letzte Kapitel „Distance, Nearness and Geometry“ adressiert abstrakte Geometrie und insbesondere Topologie. Die zweite Auflage von ,Theoretical Geography“ (Bunge, 
1973) wurde erweitert um zwei Kapitel, die 1966 als „Appendix to Theoretical Geography“ (Bunge, 1966a) erschienen. In diesen widmet sich Bunge räumlichen Beziehungen und Verteilungsmustern und diskutiert dies vor dem Hintergrund einer jüngeren Debatte in der Geographie.

Neben Christaller, dem Bunge das Buch widmet, ist wesentlicher Bezugspunkt für Bunge und sowohl die erste als auch letzte Referenz Schaefers posthum erschienener Aufsatz „Exceptionalism in Geography“ (Schaefer, 1953). Schaefers Text ist nicht nur einer der schärfsten Angriffe auf die klassische Geographie sondern auch einer der ersten Versuche, eine neue wissenschaftstheoretische Grundlegung für die Geographie zu finden. Zudem legt er eine wichtige Grundlage für eine Geographie, die auf die Geometrie von Raumgesetzen fokussiert und liefert damit die Grundlage dafür, die epistemischen Dinge der Geographie in anderer Weise zu visualisieren. Entsprechend hat dieser Text wie kaum ein anderer in der Geographie eine erbittere Diskussion zwischen den beiden Lagern ausgelöst (siehe auch die Replik von Hartshorne, 1955; Hartshorne, 1988; Martin, 1989). Der Aufsatz des vor politischer Verfolgung aus Deutschland emigrierten Schaefer, verortet sich in der Tradition des logischen Positivismus und des Wiener Kreises und war damit einer der ersten Texte, der eine Rhetorik und Rationalität von Wissenschaftstheorien des 20. Jahrhunderts in die Geographie einführte. Die posthume Veröffentlichung des Textes wurde von Gustav Bergmann, Wissenschaftstheoretiker, Freund von Schaefer und emigriertes Mitglied des Wiener Kreises organisiert (Bunge, 1968b).

Schaefer kritisiert das, was er als die auf Kant zurückgehende exzeptionalistische Grundlegung der Geographie bezeichnet und für die insbesondere Hettner und Hartshorne stünden. Mit diesem Begriff adressiert Schaefer Kants Vorstellung Geographie und Geschichtswissenschaft seien im Feld der Wissenschaften dahingehend einzigartig, dass sie die Wissenschaften der Beschreibung und der Erkenntnis des Raumes und der Zeit seien (Kant, 1923:159ff). Dies, so Schaefer, habe es verunmöglicht, dass sich die Geographie an modernen Wissenschaftstheorien ausrichtet und damit legitimer Teil der modernen und spezialisierten Wissenschaften geworden sei. Im Gegensatz $\mathrm{zu}$ anderen Wissenschaften sei die Geographie zu einer Disziplin geworden, deren Fluchtpunkt in der Beschreibung einzelner Raumausschnitte und Regionen, nicht in Systematik und der Suche nach Gesetzen gesehen wurde. Eine Beschreibung, so Schaefer und in Übereinstimmung mit einem Versprechen von Einheitswissenschaft, sei aber in keiner Weise Ziel von Wissenschaft, sondern allenfalls deren Ausgangspunkt und dies gälte entgegen der Meinung von Historikern und Geographen für alle Disziplinen. Ein Verharren in der Beschreibung sei, so Schaefer, kein Indikator für eine exzeptionalistische Wissenschaft, sondern vielmehr für ein vorwissenschaftliches und naives Stadium einer Disziplin (Schaefer, 1953). Wenn Raumausschnitte als einzigartig erscheinen und dem wird von Schaefer und Bunge nicht widersprochen (Bunge nutzt später die schöne Formulierung ,,regions are sort of unique“, Bunge, 1974), so ist dies interessant für alltägliche Erfahrungen. Dies sei aber nicht Gegenstand von Wissenschaft und Theorie (Bunge, 1966a:214). Wissenschaft, so schreibt Bunge in einem späteren Text, sei die Suche nach Wahrheit, nicht die nach reinen und unverbundenen Fakten. Wissenschaft sei Verallgemeinerung und nicht Genauigkeit (Bunge, 1971:o.A.) und damit sei sie ,diametrically opposed to the doctrine of uniqueness. It is willing to sacrifice the extreme accuracy obtainable under the uniqueness point of view in order to gain the efficiencies of generalization“" (Bunge, 1973:9).

So wie alle Wissenschaften nach Regelmäßigkeiten, Gesetzen und damit nach wiederkehrenden Mustern suchen, so müsse die Geographie als eine Raumwissenschaft nach den Raummustern und Raumgesetzen suchen, welche den geographischen Phänomenen zugrunde liegen. Diese räumlichen Muster und Gesetze sind für Schaefer in erster Linie morphologische Gesetze. ,Geography is essentially morphological“ (Schaefer, 1953:243). Dieser morphologische Charakter der Geographie, findet seinen Ausdruck insbesondere in Karten und kartographischen Darstellungen. Verbunden ist dies mit einer antihistorischen Geste, welche gegen die historisch-genetische Betrachtung der traditionellen Geographie die Geographie als Wissenschaft des Synchronen und der Kopräsenz begreift (Schaefer, 1953:243ff).

Bunge, der sich als kompromissloser (politischer und wissenschaftlicher) Verteidiger Schaefers versteht (Bunge, 1968b), schließt an diese Vorstellung an, Geographie sei eine Wissenschaft räumlicher Muster. Dies führt Bunge und andere zur naheliegenden Engführung von Geographie und Geometrie. Geometrie, als die ,language of spatial form“ (Harvey, 1969:191), sei Instrument zur Beschreibung geographischer Muster und eine Analyse dieser Muster sei Mittel zur Konstruktion von Theorien und Gesetzen. Diese Idee, Geographie als Geometrie zu bestimmen, ist in radikaler Weise damit verbunden, die Geographie mit Visuellem zu verknüpfen, sind geographische Gesetze dann doch in Form geometrischer Formen sichtbar, beschreibbar und darstellbar (Bunge, 1968c:31). Warntz bringt dies auf den Punkt, wenn er schreibt, ,Today geographers are taking the geo in geometry literally“ (Warntz, 1965:2).

Diese Engführung der Geographie mit Geometrie als einer stark räumlichen und zugleich stark visuellen Form der Mathematik, macht aus „Theoretical Geography“ ein Buch, dessen Argumentation in starkem Maße von visuellen Zeichen getragen wird. So finden sich auf etwa 200 Buchseiten der ersten Ausgabe einschließlich Graphen und Tabellen weit über 100 Abbildungen. Diese Abbildungen schöpfen aus einer Vielzahl von Genres. Neben eigenen Darstellungen finden sich Bildzitate aus Arbeiten von Autoren wie Lösch, Hägerstrand und Robinson, aber auch Karten und Kartogramme aus Politik und Medien. Die 27 Abbildungen des Kapitels zur Metakartographie beispielsweise reichen von Illustrationen geometrischer Ordnungen (Abb. 1) und ihrer Be- 
194

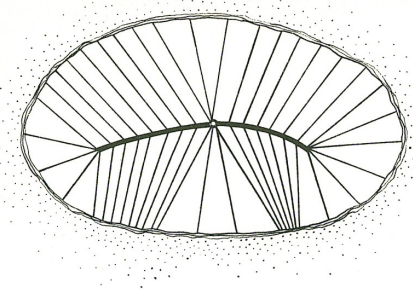

Figure 7.25. Optimally located network.

Abb. 1. Abbildung nach William Prager (Bunge, 1962:194).

rechnung über Kartogramme (Abb. 3, Abb. 1), historische Karten, Projektionen und Beispiele für Generalisierung bis zu Fragen von Grundkarten, Problemen der Kartenrezeption und schließlich zu mathematischen Übersetzungen in binäre Ordnungen. (Abb. 4). Auffällig ist dabei die Abwesenheit fotografischer Darstellungen, der sicherlich wichtigsten Visualisierungsform länderkundlicher und regionalistischer Geographien.

Die für einen geographischen Text außerordentlich abstrakt formulierte Arbeit, zieht ein weites Feld geographischer Themen heran um die formulierten Argumente zu unterstützen und setzt neben Darstellungen mit unmittelbarem inhaltlichem Bezug zur Geographie eine Reihe allgemeiner geometrischer Figuren und Darstellungen ein, um die Übersetzung realräumlicher Regionalisierungen in mathematische Beschreibbarkeit zu plausibilisieren. So finden sich bei Bunge einerseits Visualisierungen von migrations-, wirtschafts- verkehrs- oder siedlungsgeographischen Themen aus quantitativ-theoretischen Arbeiten (Abb. 2), visuelle Bearbeitungen des „Travelling Salesman“ (Bunge, 1962:158) oder Kartenanamorphoten zu Raumüberwindungskosten in Seattle (Abb. 3). Andererseits ist der Text reich an Visualisierungen von „optimally located networks“ (Abb. 1), der ,Illustration of the ordering property of Euclidean space“ (Bunge, 1962:63) oder der „Areal diffusion of a hypothetical innovation“ (Bunge, 1962:118).

\section{Mustererkennung}

Mit dem Denken der frühen quantitativ-theoretischen Geographie, für das Bunges ,Theoretical Geography“ hier als paradigmatischer Text steht - gerade auch weil der Text zahlreiche Visualisierungen anderer Autoren dieser Perspektive reproduziert und zusammenführt - , setzten sich Diagramme, Visualisierungen von Raummustern und Modelle geographischer Gesetze als wesentliche Formen geographischer Visualisierung durch. Wenn Visualisierungen vorher meist zur Unterstützung der - oftmals bildhaften - sprachlichen Schilderung räumlicher Individuen dienten, so sind die Darstellungen bei Bunge von einer radikalen Vereinfachung ge-

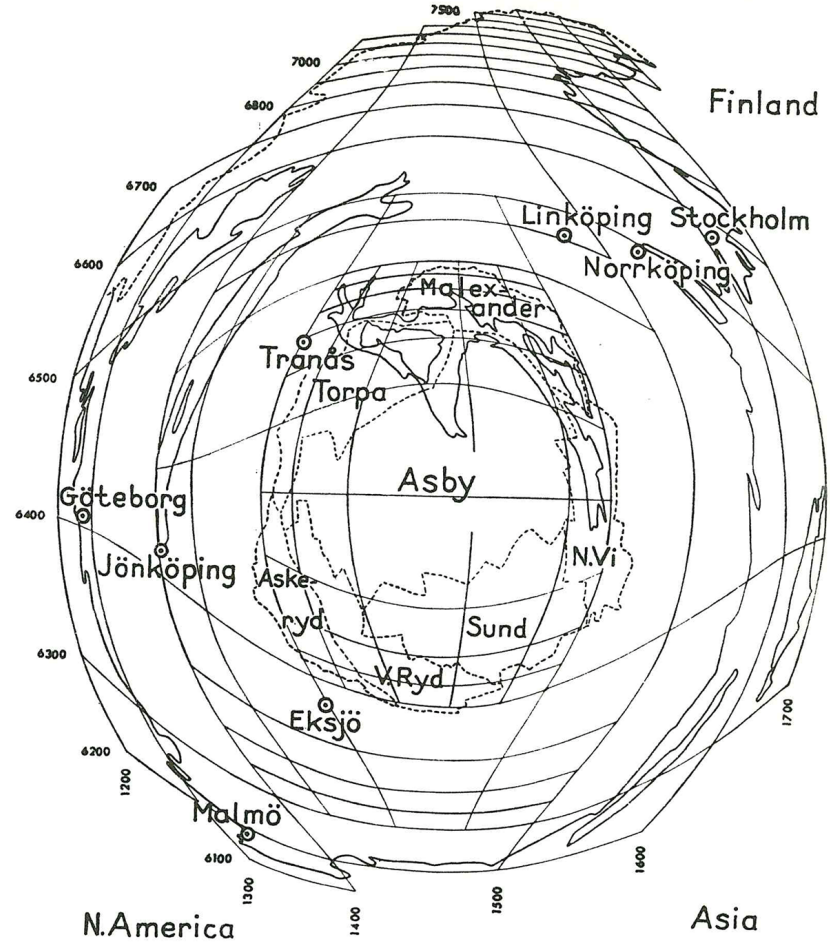

Figure 2.12. Map with azimuthal logarithmic distance scale.

Abb. 2. Reproduktion von Hägerstands „Location of more important places in the migration field of Asby“ (1957) in „Theoretical Geography“ mit veränderter Bildunterschrift (Bunge, 1962:54).

prägt. Sie erscheinen im Modus dessen, was Porter als „thin description“ bezeichnet hat (Porter, 2012:211), jenem Ideal moderner Wissenschaften von Berechenbarkeit, Klarheit und Distanz. Die Bilder dieser Geographie sind nicht nur abstrakter und erscheinen einfacher als die Fotografien, Zeichnungen und Schilderungen länderkundlicher Arbeiten, sondern mit ihnen gehen ganz andere Vorstellungen von der Funktion von Visualisierung für die wissenschaftliche Erkenntnisproduktion einher. In ihnen zeigen sich die Obsession der frühen quantitativ-theoretischen Geographie mit Fragen der Ordnung und Symmetrie ebenso wie eine Betonung eines relativen Raumbegriffs, ein Anschluss an Modelle und Metaphern aus Physik und Ökonomie und eine Ausrichtung auf Steuerung, Prognostik und Planung. Als ein eklatantes Beispiel für derartige Visualisierungen kann Christallers Hexagonalgeometrie des Systems der zentralen Orte stehen, das als Modell, Metapher, Karte, Diagramm und Instrument zugleich funktionierte und von Autoren wie Bunge zu einer Ikone der neuen Geographie überhöht wurde (Michel, 2016b).

Unterstützt werden diese Gleichgewichtstheorien und die Vorstellung harmonischer geographischer Ordnung durch Analogieschlüsse zu anderen Disziplinen, die vielfach auf zeitgenössischen geographischen Aktualisierungen der Sozialphysik aufbauen (Stewart, 1947:29f; Stewart und Warntz, 1958). Wie bei anderen Autoren der quantitativ- 


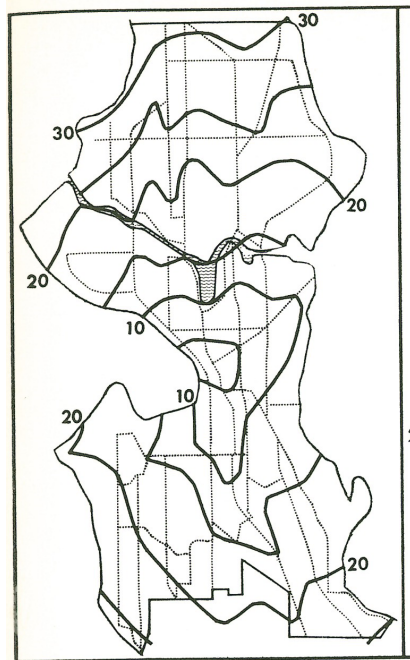

Figure 2.13. Peak hour travel time from central Seattle, five minute intervals.

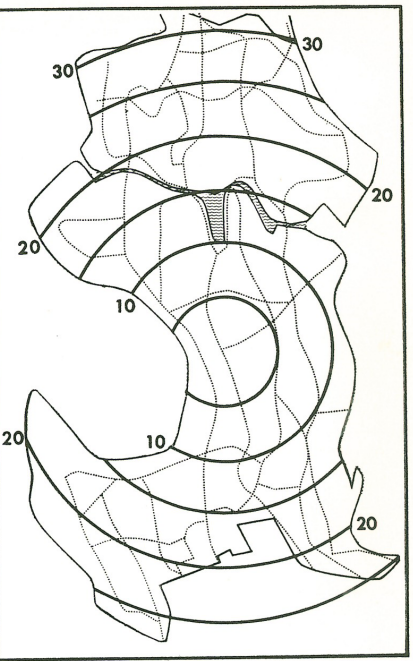

Figure 2.14. "Real" time distances from downtown Seattle, five minute intervals.

Abb. 3. Bunge (1962:55).

theoretischen Geographie, so ziehen sich morphologische Analogien zwischen sozialen und natürlichen Prozessen durch das Buch, die in erheblichem Maße dazu beitragen, die Aussagen mit Autorität auszustatten. Seien es Analogien zwischen der Veränderung von Autobahnrouten und Flussläufen in Folge von Kapazitätsüberschreitungen (Abbildungen bei: Bunge, 1962:29f), die Verteilung von Vulkanen in einer Gebirgskette, Tankstellen an einer Straße oder die Referenz auf Gravitationsmodelle, rank size rule oder Bewegungsgesetze von Dingen ,such as water, people, electricity, and goods" (Bunge, 1962:109). Am Beispiel des Flusslaufs des Mississippis und des Highways 99 werden Fragen der Geomorphologie oder des Bodenmarktes einer Perspektive auf räumliche Bewegungsgesetze untergeordnet. Geometrie wird somit zur neuen Integrationsmaschine der Geographie, die die Differenzen zwischen Humangeographie und physischer Geographie aufhebt und beide zu Gunsten räumlicher Muster zusammenfügt. „Whatever the type of movement, it leaves its mark on the face of the earth. That is, it produces the geometry" (Bunge, 1962:200).

Damit wird deutlich, wie diese neue Geographie einen neuen Begriff von Raum ins Zentrum rückt, einen Raum, der das relationale Ergebnis von Prozessen der Anordnung und der Bewegungen ist und eben nicht dem vielgescholtenen altgeographischen Container entspricht. Im kybernetischen Denken der quantitativ-theoretischen Geographie findet eine Privilegierung von Prozess gegenüber Substanz statt (Halpern, 2014:44), es geht dieser Geographie nicht um die Darstellung von Dingen, sondern von Relationen und Verhältnissen zwischen Dingen. Ordnung und Struktur, so Haggett, hängen nicht an der Geometrie des Objekts, das wir

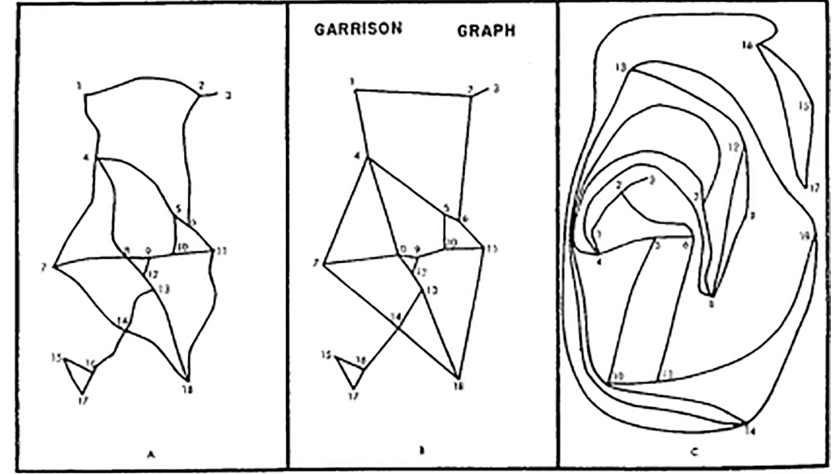

Figure 2.10. Maps and graphs of transportation routes.

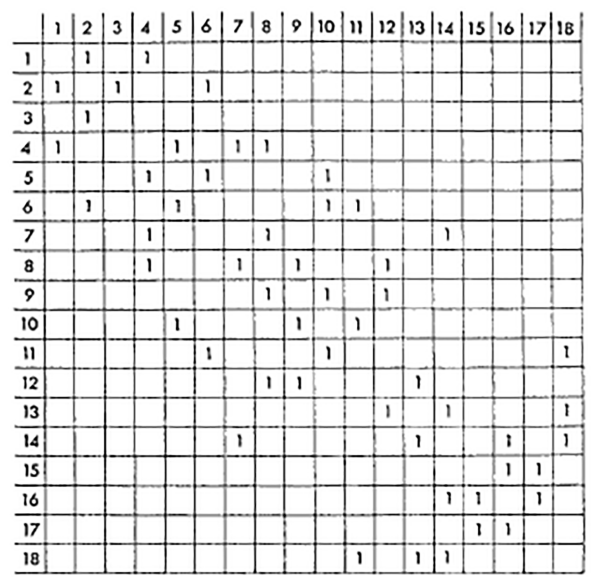

Figure 2.11. Table eqquivalent to maps and graphs in Figure 10.

Abb. 4. Garrison Graph (Bunge, 1962:53).

sehen, sondern an der Geometrie des relationalen Beziehungsgeflechts, in das es eingesetzt wird (Haggett, 1965:2). Hieraus resultiert eine Zentrierung der Geographie auf Arrangements, die als relativer Raum beschreibbar sind und damit auf solche, die in ganz anderer Weise mathematisch übersetz- und transformierbar sind, in denen metrische $\mathrm{Di}$ stanzen nur eine unter vielen möglichen Dimensionen sind. Beispielhaft hierfür sind die von Bunge zitierte Azimutalprojektion von Hägerstrand (Abb. 2) oder die Darstellung der Zeitdistanzen zum Zentrum Seattles (Abb. 3). Raum in dieser Weise als ein relationales Gefüge aus Vektoren und damit mathematisch zu denken, kartographische Transformationen als Instrumente zur Erkenntnisgenerierung und nicht der Abbildung einzusetzen und Wissen als Daten zu begreifen, ließ sich mit dem alten Paradigma der Geographie schlecht vereinbaren.

Ausgehend von einem solchen Denken in Optima und Gleichgewichten sowie einem Glauben an relativ strenge und eindeutige Theorien und Gesetze, lassen sich Forderungen nach Steuerung, Planung und Programmierbarkeit formulieren, nach einer Verbesserung schlechter Muster. Eine geographische Theorie zu haben, bedeutet hier eine Entwick- 
lung prognostizieren und damit steuern zu können (Bunge, 1962:7). Ein geographisches Phänomen zu erklären sei logisch äquivalent dazu, es vorherzusagen, und so spricht Bunge in einem Supplement zu "Theoretical Geography" beständig von „predictive or theoretical geography“ (Bunge, 1964:2). Damit dreht Bunge die zeitliche Perspektive der Geographie. Aus historisch-genetischer Beschreibung werden Prognostik und Planung geographischer Systeme. „Geography is the science of location. Regional geography classifies locations and theoretical geography predicts them" (Bunge, 1962:195).

Geographische Visualisierungen verschieben sich in dieser Geographie im Kontinuum zwischen Tabelle-DiagrammKarte-Bild-Spiegelbild (Günzel und Nowak, 2012) in Richtung des Diagramms, handelt es sich bei diesen abstrakten geometrischen Formen und Mustern doch um logische und berechnete Bilder. Sie produzieren etwas, das vorher nicht da war, machen Gesetze und Strukturen durch Visualisierung erkennbar und stellen Relationen her. Im Modus ,dünner Beschreibung“ wird Landschaft zu einem ,,set of coded topographic features, ,grounded ' by the digital logic of the grid" (Farish, 2010:144) Karten werden nicht mehr begriffen als etwas, das ein Territorium darstellt und inventarisiert, sondern als etwas, das Raum konstituiert, einen Raum, der Effekt von einer in Vektoren und geometrische Formen zerlegbaren Realität ist.

Visualisierungen dienen Bunge in „Theoretical Geography“ dazu, die oben genannten Übergänge und Übersetzungen zwischen kartographischer Rationalität der Geographie und Mathematik zu plausibilisieren. Während Mathematik dabei auch für die Geographie als am umfassendsten und flexibelsten begriffen wird, so gilt das Hauptinteresse von Bunge in „Theoretical Geography“ einer mathematisierten und damit verwissenschaftlichten Kartographie (Bunge, 1962:71), die eine Zwischenposition hält. Während geometrische Visualisierungen für einige ein Schritt hin zu einer reineren Form mathematischen Denkens in der Geographie sind (Warntz, 1957:420), liegt für Bunge in dieser sprichwörtlichen Abbildbarkeit der Geographie in geometrischen Formen ein wesentliches Moment einer geographischen Wissenschaft (Bunge, 1973:xiv). Wenn andere Disziplinen topologische Darstellungen verwenden um Beziehungen zwischen Elementen graphisch anschaulich zu machen, so kann die Geographie dies wörtlich nehmen, oder wie Bunge, Warntz paraphrasierend, schreibt ,We literally draw Venn diagrams on the earth's surface“ (Bunge, 1966b:256; Warntz, 1965:1ff).

\section{Pre-thinking GIS}

Die Übersetzung von geographischen Phänomenen oder gar „Landschaft“ in Polygone und Vektoren, in Mengendiagramme und Raummodelle mag heute wenig radikal erscheinen und nicht die Rede von einer wissenschaftlichen Revolution erlauben. Dennoch liegt hierin eines der wesentlichen Momente quantitativ-theoretischen Denkens, das eben nicht einfach und nicht in erster Linie eine Hinwendung zu Zahlen und statistischen Verfahren war. Vielmehr zeigt sich bei Bunge eine neue Vorstellung von Kartographie und geographischer Visualisierung an, wie sie in den späten 1950er Jahren von einigen Autoren entworfen wurde.

Es ist dies ein Denken von geographischen Informationen als System bzw. ein Denken, das geographisches Wissen buchstäblich als ein System geographischer Informationen begreift. Sowohl der Begriff des Systems wie auch der Begriff der Information sind hier Begriffe, die eng verbunden mit zeitgenössischem kybernetischen Denken sind, das sowohl die frühe Systemtheorie wie auch eine mathematische Formulierung von „Informationen“ ermöglichte (Poore und Chrisman, 2006). Diese Übersetzung von Raum in Geometrien, die Verwandlung von Landschaft in berechenbare und transformierbare Formen legt die konzeptionelle Grundlage für das, was wenig später als GIS entsteht. Und während die technischen Möglichkeiten des einen IBM 650 der University of Washington in Seattle mit seinen 2 Kilobyte großen Speicher (Barnes, 2004) stark beschränkt waren und manche Autoren eher frustriert von den realen Möglichkeiten dieser Maschine waren (Berry, 1966:1f), sind die epistemischen Implikationen einer solchen Übersetzungsleistung für den weiteren Verlauf der Geographie weitreichend. ${ }^{4}$

Bunge selbst hat sich in den folgenden Jahren nur noch wenig mit mathematischer Kartographie beschäftigt und sich von der Universität ab und dem politischen Aktivismus zugewandt. „Theoretical Geography“ muss aber als Text gelesen werden, der Diskussionen verdichtet und synthetisiert, die Ende der 1950er Jahre an einem Ort wie der University of Washington in Seattle virulent waren (Bunge, 1962:vii). Ein Jahr nach Bunge reichten dort Tobler (1961) und Getis (1961) ihre Dissertationen ein, kurz zuvor hatte Garrison eine Übersetzung von Netzwerken und Graphen in digitale Matrizen entworfen (Abb. 4) und wenig später beginnt Berry, der zwei Jahre zuvor bei Garrison promoviert hatte, Geographie als ,geographic matrix“ zu denken und visualisieren eine klare Vorwegnahme einer Logik von GIS-Datenbanken (Berry, 1964).

Während sich Bunge hauptsächlich mit methodologischen Fragen beschäftigte und hierbei in erheblichem Maße auf Karten zurückgriff, sind insbesondere Beiträge von Tobler maßgeblich für ein neues kartographisches Denken und Bunge nimmt vielfach auf dessen unveröffentlichte Arbeiten Bezug. Bereits in den 1950er Jahren legte Tobler eine Grundlage für eine computergestützte Kartographie und das, was als GIS einen über die Geographie hinausgehenden Wandel der

\footnotetext{
${ }^{4}$ In gewisser Weise gilt dies auch für die neue Perspektive auf das Lesen und die Nutzung von Karten, wie sie in Robinsons Karten Kommunikationsmodell zum Ausdruck kommt und das genau jener Logik der Suche störungsfreier Kommunikation in Systemen folgt (Robinson und Bartz Petchenik, 1977).
} 
Geovisualisierung einleiten sollte. Im Anschluss an das Studium der Geographie arbeitete Tobler in den 1950er Jahren für einen Ableger der RAND Corporation an der Entwicklung kartographischer Darstellungsverfahren im Rahmen des SAGE-Projektes. Dieses Projekt des militärisch-industriellakademischen Komplexes zielte auf die Entwicklung automatisierter Verfahren zur Erkennung von Interkontinentalraketen mittels gekoppelter Radarsysteme (Barnes, 2008). Daran anschließend befasst sich Tobler in ,Automation and Cartography“ erstmals mit den neuen Möglichkeiten des Computers für die Geographie und begreift aus dieser Rationalität heraus Karten als ,complex data-processing system[s]“. Als solche seien sie Instrumente der Datenspeicherung wie auch der Datenausgabe (Tobler, 1959:526ff). Als Ziel kartographischer Darstellung galt nun nicht mehr alleine die räumliche Inventarisierung von räumlicher Elementen, sondern die Entwicklung geographischer Theorien mittels kartographischer Visualisierungen und kartographischer Transformationen (Clarke und Cloud, 2000:196). Dabei wurde betont, dass die Karte nur noch eine, wenn auch eine für die Geographie bedeutsame, Form der Speicherung, Verarbeitung und Darstellung von Geoinformationen bilde und dass letztere so sie in eine mathematische Sprache gebracht seien - in eine Vielzahlt anderer Speicherungs-, Verarbeitungs- und Darstellungssysteme transferiert werden könnten (Bunge, 1962:53; Haggett und Chorley, 1969:3ff).

Zugleich enthält der Text eine Karte, eine automatisch produzierte und transformierbare, wenn auch visuell wenig spektakuläre „Map of the United States drawn directly by machine from a deck of 353 punched cards. Plotting time approximately 15 min.“ (Tobler, 1959:532). Automatisierte und analytische Kartographie zielten aber letztlich auf etwas anderes als alleine eine vereinfachte und beschleunigte Produktion von Karten mittels archivierter Sätze von Lochkarten $\mathrm{ab}$, zumal diese Daten erst auf der Basis bestehender analoger Karten gewonnen worden waren. Stärker als bisher fungierte die Karte nun als ein Medium, das Wissen generiert. In Toblers (1961) eingereichter Dissertation ,Map Transformations of Geographical Space“ (Tobler, 1961) wurden Karten zu visuellen Denk- und Beweiswerkzeugen, die nicht repräsentierten, sondern Sichtbarkeit produzierten. Denn einerseits konnten Karten jetzt zunehmend als Schichten quantifizierter und distinkter Geodaten gedacht werden, zwischen denen sich mathematisch überprüfbare Relationen herstellen ließen - gleichgültig ob manuell oder mittels elektronischer Rechenmaschinen. Damit verschob sich der Fokus kartographischen Interesses von der Karte auf diese hinter den Karten liegenden Relationen abstrahierter Daten. Statt als künstlerisch-handwerkliche Darstellung wissenschaftlich kontrollierten Wissens über einen Raumausschnitt begriffen zu werden, wurde die Karte hier als automatisch prozessierter Output einer Datenbank von Geodaten konzeptualisiert.

Andererseits wurden metrische Distanzen, Richtungen und Flächen, deren realitätsgetreue Abbildung zentrales Ziel und größtes Problem der Kartographie war, von Tobler nur noch als eine Dimension neben anderen betrachtet. In Erweiterung älterer Überlegungen zu Kartogrammen bzw. Kartenanamorphoten wies er darauf hin, dass auch Transformationen in Richtung anderer Dimensionen wie Transportkosten, Zeit oder räumlich verteilter Quantitäten beliebiger anderer Variablen möglich seien (Abb. 2; Abb. 3).

\section{Schluss}

Die visuelle Tradition der Geographie und die Vorstellung, dass es dieser Disziplin um die im Raum sichtbaren oder zumindest sichtbar zu machenden Dinge geht, macht es plausibel, dass die Wende hin zu Mathematik und Abstraktion eine Wende hin zu Geometrien ist. Dies wurde in diesem Artikel an der Arbeit von William Bunge deutlich gemacht, der hier als ein Vordenker nicht nur quantitativ-theoretischen Denkens in der Geographie, sondern einer neuen visuellen Sprache behandelt wurde. Bunge hat in seiner Arbeit wesentliche Ansätze einer Neuformulierung der späten 1950er Jahre zusammengefasst und damit als erster den Versuch unternommen, auf der Basis zahlreicher kleinerer Arbeiten, eine kohärente neue Geographie zu entwerfen. Diese neue visuelle Sprache, so wurde in diesem Text argumentiert, machte die Verschränkung von Kartographie, Geographie und Computer in den 1960er Jahren erst denkbar. In der dominierenden Erzählung der Geschichte von GIS als einem rein technischen und technologischen Innovationsprozess (Chrisman, 2006), der in den 1960er Jahren einsetzte und letztlich durch die Fähigkeiten von Computern determiniert worden sei, hat dieser Artikel deutlich machen sollen, dass zunächst eine neue Geographie gedacht werden musste. Die klassische Geographie der Länderkunde hätte mit den technischen Möglichkeiten des Computers wenig anzufangen gewusst.

Bunge selbst hatte an dieser Entwicklung keinen Anteil mehr, dies übernahmen Autoren wie Tobler in der Geographie und wäre nicht denkbar ohne parallele Entwicklungen in anderen Disziplinen, dem Militär und staatlichen Verwaltungen, die in diesem Artikel aber ausgeblendet wurden. Nach seinem Ausscheiden aus der Universität begann er in der Mitte der 1960er Jahren das politisch-aktivistische Projekt der Detroit Geographical Expedition und die Publikation von „Fitzgerald, Geography of a Revolution“ (Bunge, 1971), einer sehr lokalen und dichten Auseinandersetzung mit Rassismus und Armut in Detroit - in dessen Zuge er gegenüber der alten Geographie und ihrem Fokus auf die lokale Einzigartigkeit eine Reihe von Zugeständnissen machte (Bunge, 1974, 1968a). Weiterhin der Karte als einem wesentlichen Moment geographischer Wissensproduktion und politischer Intervention verpflichtet, brachte ihn dies von theoretischen Arbeiten weg und hin zu einer Mischung aus Kritischer Kartographie und Aktivismus, die jedoch nicht weniger radikal war, als seine frühen Arbeiten. 
Datenverfügbarkeit. Für diesen Artikel wurden keine Datensätze genutzt.

Interessenkonflikt. Der Autor erklärt, dass kein Interessenkonflikt besteht.

Danksagung. Unterstützt durch die Fritz-Thyssen-Stiftung, herzlicher Dank an Katharina Paulus, Tim Elrick und die beiden anonymen GutachterInnen.

Edited by: Benedikt Korf

Reviewed by: two anonymous referees

\section{Literatur}

Ackerman, E.: Geographic Training, Wartime Research, and Immediate Professional Objectives, Annals of the Association of American Geographers, 35, 121-143, 1945.

Barnes, T.: Placing ideas: genius loci, heterotopia and geography's quantitative revolution, Prog. Human Geogr., 28, 565-595, 2004.

Barnes, T.: Geography's underworld: The military-industrial complex, mathematical modelling and the quantitative revolution, Geoforum, 39, 3-16, 2008.

Barnes, T.: Big Data, little History, Dialogues in Human Geography, 3, 297-302, 2013.

Barnes, T.: The odd couple: Richard Hartshorne and William Bunge, The Canadian Geographer, 60, 458-465, 2016.

Barnes, T.: A marginal man and his central contributions: the creative spaces of William ("Wild Bill") Bunge and American geography, Environ. Plan. A, https://doi.org/10.1177/0308518X17707524, online first, 2017.

Barnes, T. and Abrahamsson, C.: The Imprecise Wanderings of a Precise Idea: The Travels of Spatial Analysis, in: Jöns, H., Meusburger, P., und Heffernan, M., Mobilities of knowledge. Cham: Springer Open (Knowledge and space, volume 10), 105-121, 2017.

Barnes, T. and Heynen, N.: William W. Bunge (1971) Fitzgerald: Geography of a Revolution, Prog. Human Geogr., 35, 712-715, 2011.

Barnes, T. and Wilson, M.: Big Data, Social Physics, and Spatial Analysis: The Early Years, Big Data \& Society, 1, 1-14, 2014.

Barney, T.: Mapping the Cold War. Cartography and the Framing of America's International Power. Chapel Hill: University of North Carolina Press, 2015.

Bartels, D.: Zur wissenschaftstheoretischen Grundlegung einer Geographie des Menschen, Wiesbaden: Franz Steiner, 1968.

Berry, B.: Approaches to Regional Analysis: A Synthesis, Ann. Assoc. Am. Geogr., 54, 2-11, 1964.

Berry, B.: Essays on Commodity Flows and the Spatial Structure of the Indian Economy. Chicago: Department of Geography University of Chicago, 1966.

Berry, B. and Marble, D. (Hrsg.): Spatial Analysis. A Reader in Statistical Geography, Englewood Cliffs: Prentice-Hall, 1968.

Bunge, W.: Brief an Richard Hartshorne. Hartshorne Papers, Archives of the American Association of Geographers, Milwaukee, AGSL MSG 23 Box 351 Folder 9, 1959.
Bunge, W.: Theoretical Geography. Lund: C.W.K. Gleerup (Lund studies in geography Ser. C, 1), 1962.

Bunge, W.: Patterns of Location. Discussion Paper of the Michigan University Community of Mathematical Geographers 3. Ann Arbor: Department of Geography University of Michigan, 1964.

Bunge, W.: Appendix to Theoretical Geography. Lund: C.W.K. Gleerup (Lund studies in geography Ser. C, 6), 1966a.

Bunge, W.: Gerrymandering, Geography, and Grouping, Geogr. Rev., 56, 256-263, 1966b.

Bunge, W.: Brief an Richard Hartshorne. Hartshorne Papers, Archives of the American Association of Geographers, Milwaukee, AGSL MSG 23 Box 351 Folder 9, 1968a.

Bunge, W.: Fred K. Schaefer and the Science of Geogrpahy. Special Papers Series Paper A. Cambridge: Laboratory for Computer Graphics and Spatial Analysis. Harvard Center for Envionmental Design Studies, 1968b.

Bunge, W.: Spatial Prediction, in: The Philosophy of Maps, Herausgeber: Bunge, W., Guyot, R. K. A., Martin, R., Pattison, W., Tobler, W. R., Toulmin, S., und Warntz, W., Ann Arbor: Department of Geography University of Michigan, 31-34, 1968c.

Bunge, W.: Fitzgerald, Geography of a Revolution, Cambridge: Schenkman, 1971.

Bunge, W.: Theoretical Geography, Second Edition, Lund: C.W.K. Gleerup (Lund studies in geography Ser. C, 1), 1973.

Bunge, W.: Regions are sort of unique, Area, 6, 92-99, 1974.

Bunge, W.: Fitzgerald. Geography of a Revolution, Athens: University of Georgia Press, 2011.

Burton, I.: Quantitative Revolution and Theoretical Geography, Can. Geogr., 7, 151-162, 1963.

Chorley, R. and Haggett, P. (Hrsg.): Models in Geography, London: Methuen, 1967.

Chrisman, N.: Charting the Unknown. How Computer Maping at Harvard Became GIS, Redlands: ESRI Press, 2006.

Clarke, K. and Cloud, J.: On the Origins of Analystical Cartography, Cartographic and Geographic Information Science, 27, 195204, 2000.

Cox, K.: Classics in human geography revisited, Bunge, W. 1962: Theoretical Geography, 75, 71-73, 2001.

Erickson, P., Klein, J. L., Daston, L., Lemov, R., Sturm, T., and Gordin, M. D.: How Reason Almost Lost Its Mind. The Strange Career of Cold War Rationality, Chicago: University of Chicago Press, 2013.

Farish, M.: The contours of America's Cold War. Minneapolis: University of Minnesota Press, 2010.

Fleck, L.: Entstehung und Entwicklung einer wissenschaftlichen Tatsache. Einführung in die Lehre vom Denkstil und Denkkollektiv. 1. Aufl. Frankfurt am Main: Suhrkamp (Wissenschaftsforschung), 1980.

Garrison, W. and Marble, D. (Hrsg.): Quantitative Geography. Evanston: Department of Geography, Northwestern University, 1967.

Getis, A.: A Theoretical and Empirical Inqury into the Spatial Structure of Retail Activities, Seattle (univ. Diss): University of Washington, 1961.

Goodchild, M. F.: Theoretical Geography (1962): William Bunge, in: Key Thinkers on Space and Place, Herausgeber: Hubbard, P. und Kitchin, R., London: SAGE, 9-16, 2011. 
Gould, P.: Geography 1957-1977: the Augean Period, Annals of the Association of American Geographers, 69, 139-151, 1979.

Günzel, S. and Nowak, L.: Das Medium Karte zwischen Bild und Diagramm, in: KartenWissen. Territoriale Räume zwischen Bild und Diagramm, Herausgeber: Günzel, S. und Nowak, L., Wiesbaden: Reichert, S. 1-32, 2012.

Haggett, P.: Locational Analysis in Human Geography, 2. Aufl. London: Arnold, 1965.

Haggett, P. and Chorley, R.: Network Analysis in Geography, London: Edward Arnold, 1969.

Hagner, M.: Bilder der Kybernetik. Diagramm und Anthropologie, Schaltung und Nervensystem, in: Konstruierte Sichtbarkeiten. Wissenschafts- und technikbilder seit der Frühen Neuzeit, Herausgeber: Heßler, M., München: Wilhelm Fink, 383-404, 2006.

Halpern, O.: Beautiful data. A history of vision and reason since 1945, Durham, NC: Duke University Press (Experimental futures), 2014.

Hanson, S.: "Never Question the Assumptions" and Other Science from the Revolution, Urban Geography, 14, 552-556, 1993.

Hartshorne, R.: Exceptionalism in geography reexamined. Annals of the Association of American Geographers, Annals of the Association of American Geographers, 45, 205-244, 1955.

Hartshorne, R.: Hettner's Exceptionalism - Fact or Fiction? Hartshorne Papers, Archives of the American Association of Geographers, Milwaukee, AGSL MSG 23 Box 353 Folder 11, 1988.

Harvey, D.: Explanation in Geography, London: Edward Arnold, 1969.

Isard, W.: Location and Space-Economy, A General Theory Relating to Industrial Location, Market Areas, Land Use, Trade, and Urban Structure, New York: John Wiley \& Sons, 1956.

Isard, W.: Methods of Regional Analysis: an Introduction to Regional Science, Cambridge: MIT Press, 1960.

Kant, I.: Physisiche Geographie. Erster Band, in: Immanuel Kant: Gesammelte Schriften, Band IX, Berlin: Walter de Gruyter, 151308, 1923.

Kuhn, T. S.: Die Struktur wissenschaftlicher Revolutionen, Frankfurt am Main: Suhrkamp, 1973.

Livingstone, D. N.: The geographical tradition. Episodes in the history of a contested enterprise, Oxford: Blackwell, 1992.

Martin, G. J.: The Nature of Geography and the SchaeferHartshorne Debate, in: Reflections on Richard Hartshorne's The nature of geography, Herausgeber: Nicholas Entrikin, J. und Brunn, S., Washington, DC: Association of American Geographers (Occasional publications of the Association of American Geographers, 1), 69-88, 1989.

Merrifiled, A.: Bunge's Fitzgerald: Geography of Revolution as geography classic?, Prog. Human Geogr., 35, 715-717, 2011.

Michel, B.: Der Geographische Blick. Überlegungen zu einer Wissenschaftsgeschichte geographischer Visualitätsregime, Geogr. Z., 101, 20-35, 2013.

Michel, B.: Geographische Visualitätsregime zwischen Länderkunde und Quantitativer Revolution, in: Visuelle Geographien, Herausgeber: Schlottmann, A. und Miggelbrink, J., Bielefeld: Transcript, 209-224, 2015.
Michel, B.: Seeing Spatial Structures. On the Role of Visual Material in the Making of the Early Quantitative Revolution in Geography, Geografiska Annaler B, 98, 189-203, 2016a.

Michel, B.: Strukturen Sehen. Über die Karriere eines Hexagons in der quantitativen Revolution, Geogr. Helv., 71, 303-317, https://doi.org/10.5194/gh-71-303-2016, 2016b.

Mountz, A.: Reflections on Bill Bunge's Fitzgerald: Geography ofa Revolution, Prog. Human Geogr., 25, 717-720, 2011.

Murphy, D.: Last Futures. Nature, Technology and the End of Architcture, London: verso, 2016.

Poore, B. S. and Chrisman, N.: Order from Noise: Toward a Social Theory of Geographic Informationm, in: Annals of the Association of American Geographers, 96, 508-523, 2006.

Porter, T. M.: Thin Description. Surface and Depth in Science and Science Studies, Osiris, 27, 209-226, 2012.

Rankin, W.: After the Map. Cartography, Navigation, and the Transformation of Territory in the Twentieth Century. Chicago: University of Chicago Press, 2016.

Rheinberger, H.-J.: Experimentalsysteme und epistemische Dinge. Eine Geschichte der Proteinsynthese im Reagenzglas. 2. Aufl. Göttingen: Wallstein-Verl (Wissenschaftsgeschichte), 2002.

Robinson, A. H. and Bartz Petchenik, B.: The Map As A Communication System, Cartographica, 14, 92-110, 1977.

Schaefer, F. K.: Exceptionalism in Geography: A Methodological Examination, Annals of the Association of American Geographers, 43, 226-249, 1953.

Smith, N.: "Academic War Over the Field of Geography": The Elimination of Geography at Harvard, 1947-1951, Annals of the Association of American Geographers, 77, 155-172, 1987.

Solovey, M. and Cravens, H.: Cold War social science. Knowledge production, liberal democracy, and human nature. 1. ed. New York, NY: Palgrave Macmillan, 2012.

Stewart, J. Q.: Empirical Mathematical Rules concerning the Distribution and Equilibrium of Population, Geogr. Rev., 37, 461-485, 1947.

Stewart, J. Q. and Warntz, W.: Physics of Population Distribution, J. Reg. Sci., 1, 99-123, 1958.

Tobler, W. R.: Automation and Cartography, Geogr. Rev., 49, 526534, 1959.

Tobler, W. R.: Map Transformations of Geographical Space. Seattle (univ. Diss): University of Washington, 1961.

Warntz, W.: Contributions toward a Macroeconomic Geography: A Review, Geogr. Rev., 47, 420-424, 1957.

Warntz, W.: A Note on Surfaces and Paths and Applications to Geographical Problems. Discussion Paper of the Michigan University Community of Mathematical Geographers 6. Ann Arbor: Department of Geography University of Michigan, 1965.

Wilson, M. W.: New lines? Enacting a social history of GIS, The Canadian Geographer, 2014. 